\title{
Embedding the dynamics of a single delay system into a feed-forward ring
}

\author{
Vladimir Klinshov ${ }^{1}$, Dmitry Shchapin ${ }^{1}$, Serhiy Yanchuk ${ }^{2}$, Matthias Wolfrum $^{3}$, Otti D'Huys ${ }^{4}$, and Vladimir Nekorkin ${ }^{1}$ \\ ${ }^{1}$ Institute of Applied Physics of the Russian Academy of Sciences, \\ 46 Ul'yanov Street, 603950, Nizhny Novgorod, Russia \\ ${ }^{2}$ Technical University of Berlin, Institute of Mathematics, \\ Straße des 17. Juni 136, 10623 Berlin, Germany \\ ${ }^{3}$ Weierstrass Institute for Applied Analysis and Stochastics, Mohrenstr. 39, 10117, Berlin, Germany and \\ ${ }^{4}$ Aston University, Department of Mathematics, B4 7ET Birmingham, United Kingdom
}

\begin{abstract}
We investigate the relation between the dynamics of a single oscillator with delayed self-feedback and a feed-forward ring of such oscillators, where each unit is coupled to its next neighbor in the same way as in the self-feedback case. We show that periodic solutions of the delayed oscillator give rise to families of rotating waves with different wave numbers in the corresponding ring. In particular, if for the single oscillator the periodic solution is resonant to the delay, it can be embedded into a ring with instantaneous couplings. We discover several cases where the stability of a periodic solution for the single unit can be related to the stability of the corresponding rotating wave in the ring. As a specific example we demonstrate how the complex bifurcation scenario of simultaneously emerging multi-jittering solutions can be transferred from a single oscillator with delayed pulse feedback to multi-jittering rotating waves in a sufficiently large ring of oscillators with instantaneous pulse coupling. Finally, we present an experimental realization of this dynamical phenomenon in a system of coupled electronic circuits of FitzHugh-Nagumo type.
\end{abstract}

\section{INTRODUCTION}

A specific feature of systems with time delayed feedback is that their phase space is infinite dimensional. In particular, for large delay times, this can lead to complex high dimensional dynamics [1-11]. Similarly, complex high dimensional dynamics can be observed in large network systems [12-15]. In this case, the presence of long feed-forward loops can play a similar role to a direct feedback with a large delay time.

In this paper, we will substantiate this heuristic correspondence and provide a detailed study of the relation between periodic orbits of oscillators with time delay and rotating waves in corresponding systems where many oscillators of this type are coupled in a feed-forward ring structure. Rings are fundamental motifs that often appear, and potentially play an important role in the dynamics of recurrent complex networks, in particular neural networks [16-20]. Moreover, the ring motifs of connections that can be singled out from more complex connectivity patterns often play an important role in the network dynamics [21-25]. The dynamics of feed-forward rings have been studied before, including rotating waves and patterns [26-28], chaotic and complex dynamics [29$31]$ and transient processes [32,33]. The influence of coupling delays has been also investigated theoretically [34-37] and experimentally [38-44].

We start from the basic observation that a periodic solution of a single oscillator with delayed feedback can be embedded into the feed-forward ring if certain relations between the delay times of the feedback oscillator and the delay in the coupled ring are satisfied. For periodic solutions with a period that is resonant, i.e. relates rationally to the delay time, the embedding is possible in a ring with instantaneous coupling, i.e. without delay. Furthermore, we discuss several cases where the stability properties of the periodic solution of the feedback oscillator and the rotating wave in the ring are related.

An important tool for this investigation is the multiscale approach to systems with large delay [11, 45, 46], where the singular limit of the delay time tending to infinity is used to obtain an asymptotic description of the stability. Note that a periodic orbit of a delay differential equation is again a solution when the delay is increased by an integer multiple of the period [47]. In this way the orbit reappears for a sequence of delay times and can be found for arbitrary large delays. A similar approach can be used not only in the case when both the feedback delay of the single oscillator and the coupling delay in the ring are large, but also in the case of instantaneous coupling in a sufficiently large ring. Another similarity between delay systems and rings was mentioned in $[27,48,49]$, where certain important features of the spectrum of steady states and periodic solutions in systems with large delay and rotating waves in large feed-forward rings are reported. In Refs. $[31,50]$, it is shown that the correlation properties of a ring with $N$ elements can be deduced from the autocorrelation of the single delayed feedback system.

A specific example for the emergence of complex dynamics in systems with delay is the recently discovered regime of multi-jittering in oscillators with delayed pulse feedback [41, 51-53]. Based on the theoretical background given in the first part of the paper, we show that this phenomenon has its counterpart in jittering rotating waves in a feed-forward pulse-coupled ring. Their appearance can even be demonstrated experimentally by a system of coupled electronic FitzHugh-Nagumo circuits.

The structure of the paper is as follows. Section I presents theoretical results on the correspondence between periodic solutions of the delayed oscillator and the ring. It also discusses the cases when the stability 
of corresponding periodic solutions is related. Further, Sec. II explains how periodic solutions of a delay feedback oscillator are embedded as rotating waves in a ring with instantaneous coupling under the condition of resonance between period and delay. In Sec. III we show how the multi-jittering bifurcation known from delayed systems, appears in feed-forward rings with instantaneous couplings. In particular, in Sec. III B, an experimental demonstration of the jittering waves is presented in a ring of electronic FitzHugh-Nagumo oscillators.

\section{PERIODIC SOLUTIONS OF A SINGLE DELAYED OSCILLATOR AND ROTATING WAVES IN A UNIDIRECTIONALLY COUPLED RING}

We start with a single oscillator with delayed feedback

$$
\frac{d x(t)}{d t}=f(x(t), x(t-\tau)),
$$

where $x \in \mathbb{R}^{d}$, and $\tau \geq 0$ is the time-delay. We assume that for $\tau=\tau_{0}$ equation (1) has a periodic solution $h(t)$ with a period $T$. It is easy to see that changing the delay, $h(t)$ reappears as a periodic solution whenever multiple of its period $T$ is added to the delay, that is, $h(t)$ is a periodic solution for all values of the delay

$$
\tau_{k}=\tau_{0}+k T, \quad k \in \mathbb{N} .
$$

Note that without loss of generality, we can assume $0 \leq$ $\tau_{0}<T$, i.e. $\tau_{0}$ is the minimal positive delay at which the given solution $h(t)$ exists, such that for $k \geq 0$ we obtain all non-negative values of the delay for which the periodic solution $h(t)$ reappears. For a detailed exposition of this reappearance phenomenon and its consequences see [27].

Now we take $N$ oscillators of the form (1), where, instead of the delayed self-feedback, we employ a delayed coupling to the neighboring oscillator:

$$
\frac{d x_{n}(t)}{d t}=f\left(x_{n}(t), x_{n-1}(t-\sigma)\right)
$$

Considering the oscillator index $n=1, \ldots, N$ modulo $N$, we obtain a unidirectionally coupled ring, where we denote the coupling delay by $\sigma$ in order to distinguish it from the feedback delay $\tau$ in the single oscillator (1). Following Refs. $[54,55]$ the periodic solution $h(t)$ of the single oscillator can be used to construct rotating waves for the ring system (3) by

$$
x_{n}(t)=h(t+n \theta),
$$

where $\theta$ is the phase lag between neighboring oscillators. In order to satisfy the periodic boundary condition in the ring, this phase lag has to be resonant to the period $T$, i.e.

$$
\theta=\theta_{M}=M T / N
$$

where $M=0, \ldots, N-1$ is the wave number in the ring. It can be easily seen that the ansatz (4) leads to a rotating wave solution of (3), if the delays in the two systems satisfy the compatibility condition

$$
\sigma=\sigma_{k, M}=\tau_{0}+k T-\theta_{M}=\tau_{k}-\theta_{M}, \quad k \in \mathbb{N} .
$$

In this way, we obtain for each fixed wave number an increasing sequence of delay times $\sigma_{k, M}$ for which the periodic solution $h(t)$ of the single oscillator with delayed feedback (1) can be embedded as a rotating wave into the unidirectionally coupled ring system (3). Note that for some choices of $M$ the first value $\sigma_{0, M}$ can be negative and the first positive delay value in the reappearance sequence is $\sigma_{1, M}$.

The described relation between a single oscillator and a feed-forward ring is illustrated in Fig. 1. In Fig. 1(a) we show the branch of periodic solutions for the Van-der-Pol oscillator with delayed feedback

$$
\frac{d^{2} x(t)}{d t^{2}}=\alpha\left(1-x(t)^{2}\right) \frac{d x(t)}{d t}-x(t)+\kappa x(t-\tau),
$$

plotting the period $T$ versus the delay time $\tau$. Such form of the bifurcation diagram is typical when the period on the branch is bounded (see more details in [47]) and it clearly leads to an increasing coexistence of periodic solutions with the growth of time-delay. Note the increasing skewness of the branch that can be explained by the period depending spacing of the reappearance sequences.

For the ring of $N$ oscillators with unidirectional coupling

$$
\frac{d^{2} x_{n}(t)}{d t^{2}}=\alpha\left(1-x_{n}(t)^{2}\right) \frac{d x_{n}(t)}{d t}-x_{n}+\kappa x_{n-1}(t-\sigma),
$$

in the case of $N=3$ we obtain three branches of rotating waves shown in Fig. 1(b). While the branch for wave number $M=0$ coincides with the periodic solution for the single oscillator, shown in panel (a), the other two branches can be obtained by a shift of $\theta_{M}=M T / N$ along the horizontal axis. The stability regions are highlighted as solid parts of the branch, unstable as dashed. The stability regions vary from branch to branch, however, they turn out to coincide for large delays. This phenomenon is explained analytically in the following section.

\section{Stability properties}

A reappearing periodic solution $h(t)$ of the delay equation (1) has typically different stability properties for different values of $k$ in the reappearance sequence (2). For large values of $k$, corresponding to large values of the delay $\tau_{k}$, the stability can be asymptotically described using the singular limit $k \rightarrow \infty$. The theoretical background has been elaborated in detail in [46, 47]. It is shown there that in this case the Floquet-spectrum consists of pseudo-continuous spectrum, converging to continuous curves, and strongly unstable spectrum, converging to 

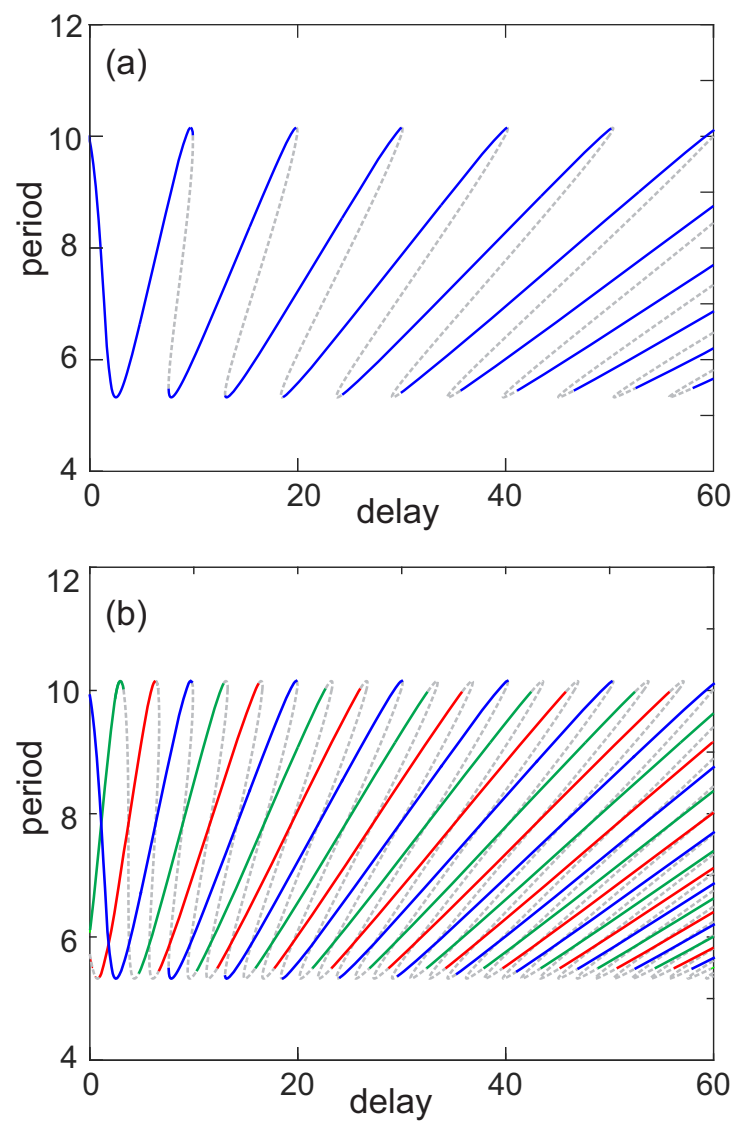

Figure 1. (a) Branch of periodic solutions for a single Vander-Pol oscillator with delayed feedback (6). Solid/dashed parts indicate stability/instability. (b) Branches of rotating waves for a feed-forward ring of $N=3$ Van-der-Pol oscillators with delayed coupling (6). Colored parts indicate stability for $M=0$ (blue), $M=1$ (red), and $M=2$ (green).

finitely many isolated points. These limiting objects can be determined from a multi-scale approach and then be used as a criterion for stability or instability for finite but sufficiently large delays $\tau_{k}$.

Based on this approach, we will show now that the periodic solution $h(t)$ of the single oscillator (1) and the corresponding rotating wave solutions (4) of the ring system (3) actually lead to the same limiting spectra and hence share the same linear stability properties, if in both cases the delay is sufficiently large, i.e. $k \rightarrow \infty$ (cf. $[54,55]$ ).

The linear stability of the solution $h(t)$ in $(1)$ is given by the following variational equation for a perturbation $\delta(t) \in \mathbb{R}^{d}:$

$$
\frac{d \delta(t)}{d t}=A(t) \delta(t)+B(t) \delta(t-\tau)
$$

where $A(t)=\partial_{1} f(h(t), h(t-\tau))$ and $B(t)=$ $\partial_{2} f(h(t), h(t-\tau))$ are the derivatives of $f$ with respect to its first and second argument, respectively. To obtain the corresponding Floquet problem, we substitute

$$
\begin{aligned}
& \delta(t)=p(t) e^{\lambda t} \text { and get } \\
& \frac{d p(t)}{d t}=(A(t)-\lambda \mathrm{Id}) p(t)+e^{-\lambda \tau} B(t) p(t-\tau),
\end{aligned}
$$

The set of complex values $\lambda$ for which Eq. (8) admits a $T$-periodic solution $p(t)$ are the characteristic exponents. Note that the coefficient matrices $A(t)$ and $B(t)$ are $T$ periodic as well. The solution $h(t)$ is stable if all characteristic exponents have negative real parts.

Similarly, the linear stability of the rotating wave solution (4) of the ring (3) is defined by the variational equation

$$
\begin{aligned}
\frac{d \delta_{n}(t)}{d t} & =\partial_{1} f(h(t+n \theta), h(t+(n-1) \theta-\sigma)) \delta_{n}(t)+ \\
& +\partial_{2} f(h(t+n \theta), h(t+(n-1) \theta-\sigma)) \delta_{n-1}(t-\sigma),
\end{aligned}
$$

where $\delta_{n}(t) \in \mathbb{R}^{d}$ is the variation of $x_{n}$ Introducing the delay compatibility condition (5) in the form $\tau=\sigma+\theta$ into the coefficient matrices, and substituting the Floquet ansatz $\delta_{n}(t)=r_{n}(t) e^{\lambda t}$, we obtain

$$
\begin{aligned}
\frac{d r_{n}(t)}{d t}= & (A(t+n \theta)-\lambda \mathrm{Id}) r_{n}(t)+ \\
& +e^{-\lambda \sigma} B(t+n \theta) r_{n-1}(t-\sigma),
\end{aligned}
$$

where $r_{n}(t)$ are $T$-periodic functions and $\lambda$ the characteristic exponents to be found. A component-wise time-shift transformation

$$
p_{n}(t)=r_{n}(t-n \theta)
$$

(see [25] for details) leads to

$$
\frac{d p_{n}(t)}{d t}=(A(t)-\lambda \mathrm{Id}) p_{n}(t)+e^{-\lambda \sigma} B(t) p_{n-1}(t-\tau) .
$$

The coupling matrix of the unidirectional ring can be diagonalized by passing to discrete Fourier modes

$$
p_{n}(t)=\sum_{m=1}^{N} \hat{p}_{m}(t) e^{2 \pi i m n / N}
$$

such that we obtain the decoupled spectral problems

$$
\frac{d \hat{p}_{m}(t)}{d t}=\left(A(t)-\lambda_{m} \mathrm{Id}\right) \hat{p}_{m}(t)+e^{-\lambda_{m} \sigma-i \psi_{m}} B(t) \hat{p}_{m}(t-\tau) .
$$

for $m=1, \ldots, N$, which are only distinguished by the phase factor

$$
\psi_{m}=2 \pi m / N
$$

and have all a similar form as the spectral problem for the single equation (8). As it is shown in Lemma 5 in [46], the characteristic exponents of a Floquet problem (8) for the single equation (1) can be obtained from a characteristic equation of the form

$$
F\left(\lambda, e^{-\lambda \tau_{k}}\right)=0 .
$$


with some analytic function $F$. This function can be used also to determine the limiting spectra for $k \rightarrow \infty$, which is given by

$$
F(\lambda, 0)=0 .
$$

for the strongly unstable spectrum and

$$
F\left(i \omega, e^{-\gamma-i \varphi}\right)=0
$$

for the asymptotic continuous spectral curves approximating weak spectrum with the limiting behavior

$$
\lambda=i \omega+\frac{\gamma}{\tau}
$$

for real $\omega$ and $\gamma$. These curves are parametrized by the extra parameter $\varphi$ in (14). For the decoupled spectral problems (11) of the ring, we obtain the same function $F(\cdot, \cdot)$ with the second argument replaced by $e^{-\lambda \sigma-i \psi_{m}}$. However, the resulting equations (13) and (14) for the limiting spectra coincide, since the phase factor $\psi_{m}$ can be absorbed into the curve parameter $\varphi$. Hence, for large delay the stability properties of the periodic solution in the single equation (8) coincides with the stability of all rotating waves independent on their wave number $M$.

\section{INSTANTANEOUS COUPLING IN THE RING OF OSCILLATORS}

So far, we studied the situation with a delay both in the single oscillator and in the unidirectionally coupled ring. However, the delay compatibility condition (5) can be satisfied also with $\sigma=0$, i.e. instantaneous coupling in the ring. This happens exactly, if for the single oscillator, we have a periodic orbit $h(t)$ with a period $T$ resonant to the delay $\tau_{0}$

$$
M_{0} T / N_{0}=\tau_{0} .
$$

Assuming that $M_{0}$ and $N_{0}$ are relative prime, the periodic solution can be embedded into a ring of minimal size $N_{0}$, and additionally for all integer multiples

$$
N_{j}=j N_{0}
$$

with the wave number being the corresponding multiple of $M_{0}$.

In Fig. 2(a) we show that a branch of periodic solutions with varying delay generically leads to the appearance of resonances of the period and the delay. In the figure the resonance conditions for $N=6$ are shown as gray lines. Their intersections with the branch of periodic solutions for a single Van-der-Pol oscillator with delayed feedback (6) correspond to resonant periodic solutions. The thin solid line indicates the stable part of the branch, while the red thick line corresponds to periodic solutions that are stable when they reappear for sufficiently large delays. As we will show below, this implies the stability of the corresponding rotating waves. In this case, stable

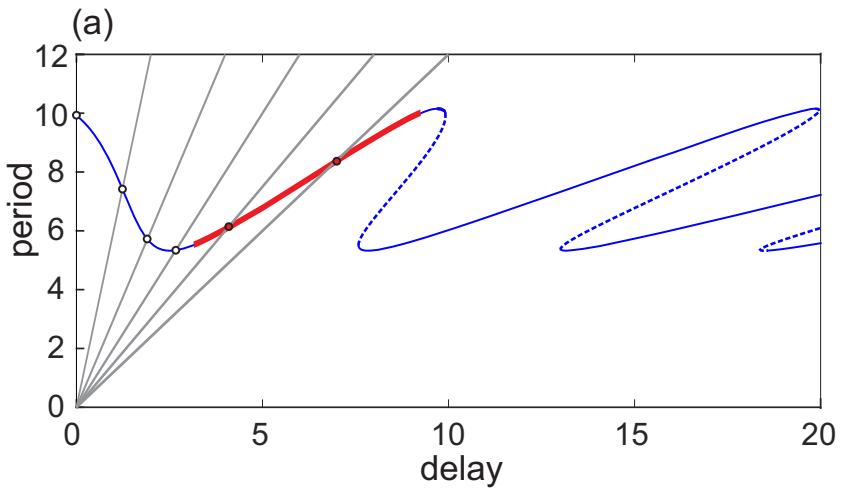

(b)

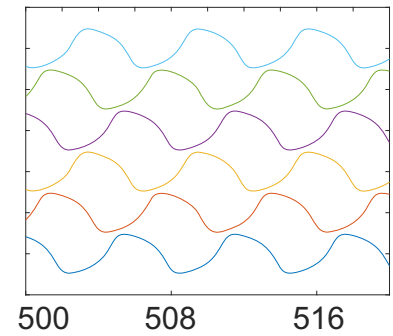

(c)

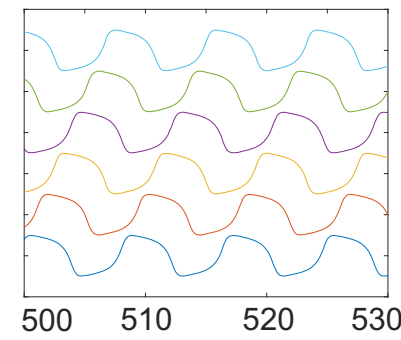

Figure 2. (a) Branch of periodic solutions for a single Vander-Pol oscillator with delayed feedback (6). Solid and dashed parts indicate stability and instability, respectively. The red thick part of the line corresponds to periodic solutions that are stable when they reappear for sufficiently large delays. Grey lines indicate the resonance conditions $\tau=M T / N$ for $N=6$ and $M=1, \ldots, 5$ and their intersections with the branch indicates resonant periodic solutions leading to rotating waves. Stable rotating waves exist for $M=4$ (b) and $M=5$ (c).

rotating waves exist only for $M=4$ and $M=5$, shown in panels (b) and (c). Note that the rotating waves with different wave numbers are unstable even although the resonant periodic orbits for some of them lie on the stable part of the branch.

As we have seen, the stability of the rotating wave may be different from the stability of the original periodic solution at $\tau_{0}$. However, we will show now that the stability of the rotating wave relates to the stability of $h(t)$ for large delays $\tau_{k}$ : If the resonant periodic solution $h(t)$ is asymptotically stable for all large enough $\tau$, then the rotating wave in the ring with no delays is stable as well.

To prove this, note that the stability of $h(t)$ for large delay implies that the branches of asymptotic continuous spectrum (15), given by (14), have negative real parts except for a single zero at $\omega=\varphi=0$ corresponding to the trivial Floquet-mode given by the shift along the periodic orbit itself (see [46], Lemma 18). Now, assume that the rotating wave is unstable, i.e. the characteristic equation

$$
F\left(\lambda, e^{-\lambda \sigma-i \psi_{m}}\right)=0,
$$

has a solution $\lambda$ with $\operatorname{Re} \lambda>0$ for $\sigma=0$. Then, by increasing $\sigma$, this solution must cross the imaginary axis 
at some point $i \Omega$ for some value $\sigma$, since for large $\sigma$ the rotating wave is stable as it is shown in Section I. According to (12) this solution satisfies

$$
F\left(i \Omega, e^{-i \Omega \sigma-i \psi_{m}}\right)=0,
$$

for some wave number $m$ of the perturbation. This contradicts to the assumption of stability of $h(t)$ for large delay, which implies that $F\left(i \omega, e^{-i \varphi}\right) \neq 0$ for all values of $\omega$ and $\varphi$ different from zero. Thus, the asymptotic stability for large delay of a periodic solution of the single oscillator (1) guarantees the stability of the corresponding rotating waves with instantaneous coupling in (3). Note that this is true for all integer multiples of the minimal ring size $N_{0}$.

Choosing a sufficiently large size $N_{j}$ of the ring, it is also possible to show the opposite: If the resonant periodic solution $h(t)$ of the single oscillator (1) is weakly unstable for large delay $\tau_{k}$, then the corresponding rotating wave is also unstable in a ring (3) of sufficiently large size $N_{j}$ and coupling delay $\sigma=0$. Indeed, in the ring with $\sigma=0$ the characteristic equation for the rotating waves takes form

$$
F\left(\lambda, e^{-i \psi_{m}}\right)=0
$$

Stability of the rotating wave would imply that all roots of (17) except for $\psi_{0}=0$ have negative real part. Since for large $N$ the phases $\psi_{m}$ densely fill the interval $[0 ; 2 \pi]$, by analyticity of $F$ all roots of

$$
F\left(\lambda, e^{-i \varphi}\right)=0
$$

with $0<\varphi<2 \pi$ have then also negative real part. At the other hand, if the periodic solution $h(t)$ of (1) is weakly unstable for long delay $\tau_{k}$, there must be some point $\Omega \neq$ 0 for which the pseudo-continuous spectrum crosses the imaginary axis, i.e. $\gamma(\Omega)=0$ and hence $F\left(i \Omega, e^{-i \varphi}\right)=0$. This contradiction proves that the rotational wave is in fact unstable.

Thus, the stability of a periodic solution of the single oscillator at large delay is a sufficient condition for the stability of the corresponding rotating wave in a ring with instantaneous coupling. For a large number $N$ of oscillators, it is also a necessary condition.

\section{JITTERING REGIMES IN A RING OF OSCILLATORS WITH INSTANTANEOUS COUPLING}

\section{A. Theoretical study of the multi-jittering}

We have shown in the previous sections that periodic solutions of a single oscillator with delayed feedback can be embedded as rotating waves in a ring with instantaneous couplings. This fact implies that regimes that have been observed in time-delayed systems, are generally expected in complex networks, that do not have necessary (a)

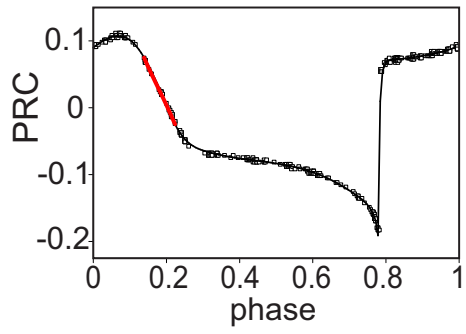

(b)

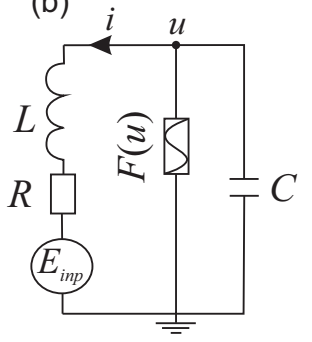

Figure 3. (a) The PRC and (b) the circuitry of the electronic FitzHugh-Nagumo oscillator.

time delays. The reverse statement is also true: dynamical phenomena observed in lattices of coupled oscillators are also expected in systems with large delays, such as chimera states [8], reservoir computing [9], Eckhaus instability [10], complex pseudo-spatial patterns [56], etc.

In this section, we concentrate on a fascinating phenomena that recently was discovered in oscillatory systems with long delays, the so-called multi-jittering instability [51]. Later similar phenomena were observed in rings with time delays [53]. Now we show that the multijittering can be observed in a ring with instantaneous couplings.

The multi-jittering was first discovered in a single oscillator with delayed pulse feedback. The minimal model is a phase oscillator, where the action of the pulse feedback is modeled with the so-called phase resetting curve (PRC) $Z(\varphi)$, which characterizes the response of the oscillator to an incoming pulse at different phases:

$$
\frac{d \varphi}{d t}=1+Z(\varphi) \sum_{t_{p}} \delta\left(t-t_{p}-\tau\right) .
$$

Here, $\varphi$ is the oscillator's phase, $Z(\varphi)$ its PRC, and $\tau$ the delay. In the absence of the feedback, the phase grows uniformly with $d \varphi / d t=1$. When the phase reaches unity, it resets to zero, and the oscillator emits a spike. The moments of spike emissions are denoted as $t_{p}$. Each spike is sent to a delay line, and it arrives to the oscillator after the delay $\tau$ causing an instantaneous phase shift $\Delta \varphi=Z(\varphi)$. Further, we select the $\operatorname{PRC} Z(\varphi)$ as in Fig. 3(a) which corresponds to the electronic FitzHughNagumo oscillator depicted in Fig. 3(b) [57, 58].

The bifurcation diagram for (18) is shown in Fig. 4 where the periods of the observed regimes are plotted versus the delay. The most common regime is the socalled regular spiking when the oscillator emits spikes with constant inter-spike interval. The stable parts of the corresponding branch are plotted by black solid line, unstable by gray dashed line.

In [51] we have shown that under quite general conditions the regular spiking regime may be destabilized via a very peculiar scenario. Namely, if the PRC function $Z(\varphi)$ has a point $\psi^{*}$ with slope $Z^{\prime}\left(\psi^{*}\right)=-1$, the so-called "multi-jitter" bifurcations take place for delay 


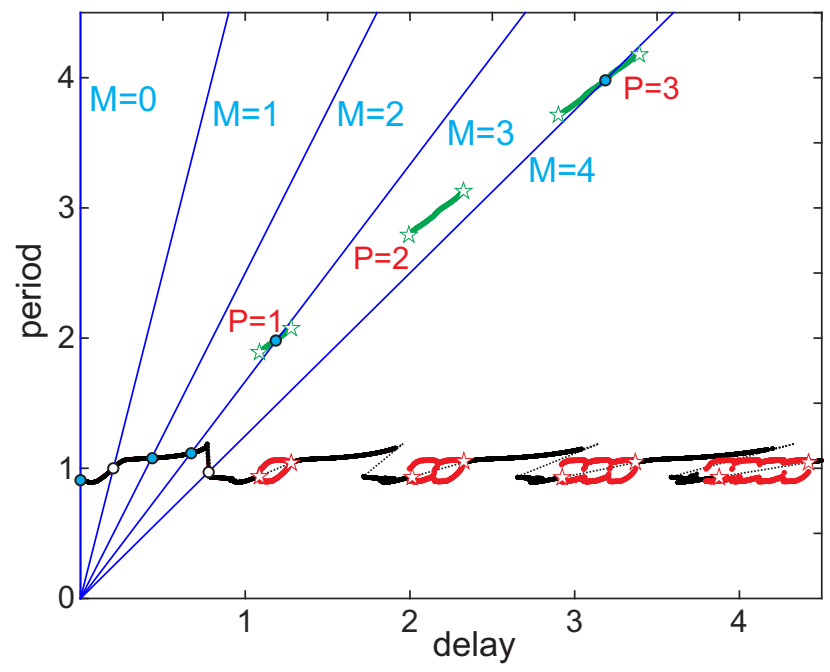

Figure 4. The bifurcation diagram of a single oscillator with pulse delayed feedback (18), period versus the delay. Black solid line corresponds to stable regular regimes, gray dashed line to unstable regular regime, green lines to jittering regimes. Red solid lines depict the inter-spike intervals that constitute the jittering regimes. Stars denote the multi-jitter bifurcation points. The bifurcation diagram is combined with a family of lines $\tau=M T / N$ with $N=5$. The intersection points correspond to rotating waves in a ring of pulse-coupled oscillators with no delays (20). Hollow points correspond to unstable, solid to stable waves.

values

$$
\tau_{J}=P\left(1-Z\left(\psi^{*}\right)\right)+\psi^{*},
$$

where $P \in \mathbb{N}$. In each of these bifurcations, the regular spiking solution destabilizes, and the so-called "jittering" solutions emerge. These regimes are characterized by a periodic sequence of non-equal inter-spike intervals. It was shown that the length of one period in the sequences equals $P+1$ inter-spike intervals, i.e. grows linearly with the delay (compare to (19)). In Fig. 4 the periods of the jittering solutions are plotted by green lines, while the inter-spike intervals constituting them are plotted by red. The latter curves branch off the regular spiking branch at the multi-jitter bifurcation points denoted by stars.

In [53], we described the jittering instability in rings of pulse delay coupled oscillators. It was shown that rotating waves in such rings may destabilize and give birth to "jittering" waves. Here we will demonstrate that the jittering waves may appear in rings without delays.

Consider a ring of oscillators with unidirectional instantaneous pulse coupling

$$
\frac{d \varphi_{n}}{d t}=1+Z\left(\varphi_{n}\right) \sum_{t_{n-1, p}} \delta\left(t-t_{n-1, p}\right)
$$

where $n=1, \ldots, N$. Here, the spikes produced by $(n-1)$ st oscillator at moments $t_{n-1, p}$ immediately arrive to the $n$-th oscillator and perturb it.
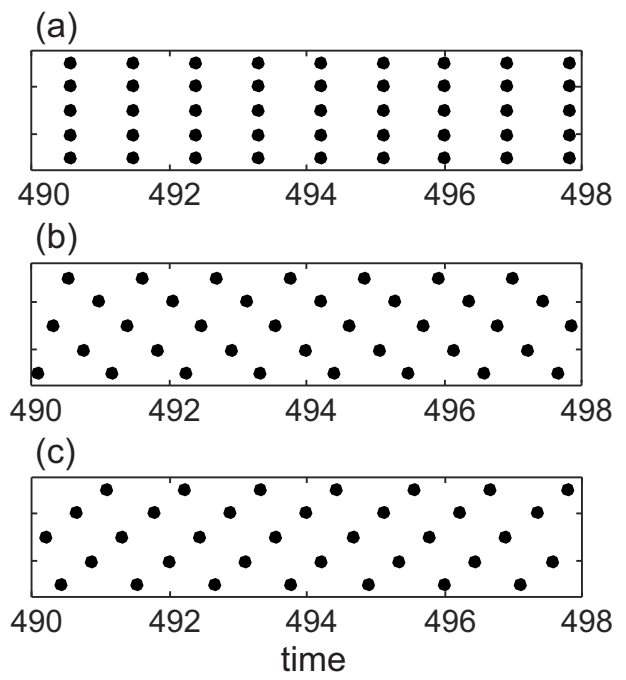

Figure 5. Regular rotating waves in (20): (a) $M=0$, (b) $M=2$ and (c) $M=3$. Points denote the moments of spikes of the oscillators.

The ring (20) consists of the same oscillators as (18), thus the periodic solutions of (18) transfer into rotating waves of (20). To obtain the rotating wave solutions, the bifurcation diagram in Fig. 4 is complemented by the family of lines $\tau=M T / N$ (similarly to Fig. 2), where $M=0,1, \ldots N-1$, and $N=5$. The points where these lines intersect the branches of the periodic solutions correspond to the rotating waves. As one can see in Fig. 4, there are 5 points where the regular spiking branch of $T(\tau)$ intersects one of the lines $\tau=M T / N$. We have checked that some of these points correspond to stable rotating waves in the ring. These points are marked by blue circles and appear on the asymptotically stable part of the branch. The other points correspond to unstable rotating waves and are marked by white circles. The stable regular waves are observed in the system for $M=0,2,3$, they are illustrated in Fig. 5(a-c).

The lines in Fig. 4 also intersect the branches of jittering solutions which implies the existence of jittering rotating waves. These waves have indeed been observed, they are illustrated in Fig. 6(a,b). In these Figures, the top panels show the moments of spikes emission by the oscillators, while the bottom panels depict the dynamics of the inter-spike intervals. The sequences of inter-spike intervals are the same for all the oscillators, although timeshifted, and consist of two distinct values (the so-called bipartite solutions). Note that the plots corresponding to the different oscillators are shifted along the vertical axis for the sake of better representation. In reality the values of the short and the long inter-spike intervals are also the same for all the oscillators. Thus, the rotating wave of inter-spike intervals propagates in the ring. 
(a)

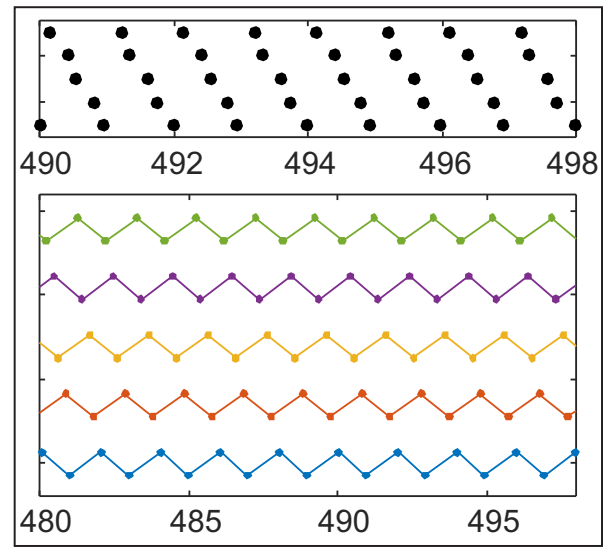

(b)

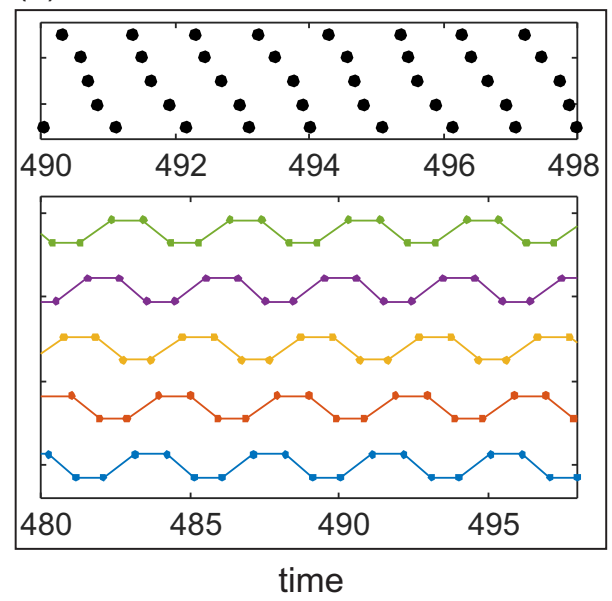

Figure 6. Two different jittering rotating waves observed in (20). The top panels depict the moments of spike generation, the bottom inter-spike intervals.

\section{B. Experimental demonstration in a ring of electronic FitzHugh-Nagumo oscillators}

We have also corroborated our theoretical results by the experiments with electronic circuits. We have experimentally studied a ring of $N=5$ electronic FitzHughNagumo oscillators with instantaneous unidirectional couplings. The circuitry of the single electronic oscillator used in the experiment is depicted in Fig. 3(b) [57, 58]. Here $u$ is the voltage on the capacitor, $i$ is the current trough the inductor, $F(u)=\alpha u\left(u-u_{0}\right)\left(u+u_{0}\right)$ is the current-voltage characteristic of the nonlinear resistor, and $E_{i n p}$ is the input signal. The parameter values were set as $R=1 \mathrm{k} \Omega, \alpha=2.02 \times 10^{-4} \Omega^{-1} \mathrm{~V}^{-2}, u_{0}=0.82 \mathrm{~V}$. The capacitance $C=5 \mathrm{nF}$ and the inductance $L=9.4 \mathrm{H}$ where set so that in the absence of coupling each oscillator emits spikes periodically with the period $T \approx 2.95 \mathrm{~ms}$ . The coupling in the ring is organized as follows: when the output voltage of any oscillator exceeds the threshold value $u_{t h}=0.84 \mathrm{~V}$, a square-shape pulse of the amplitude $A_{P}=5 \mathrm{~V}$ and duration $T_{P}=42 \mu \mathrm{s}$ is sent to the next os- (a)

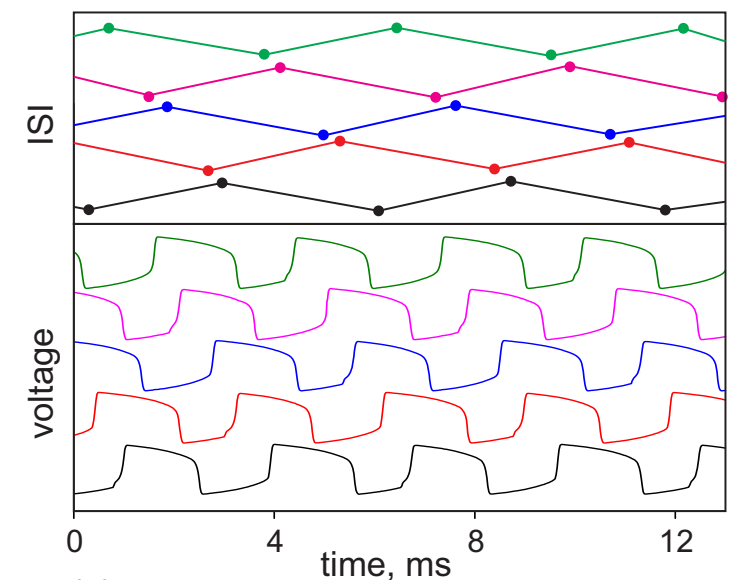

(b)

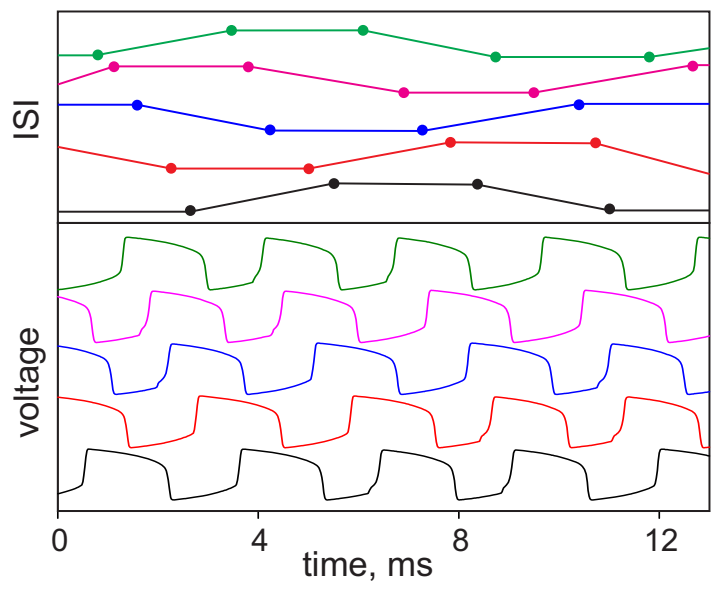

Figure 7. Two different jittering rotating waves experimentally observed in a ring of five electronic FitzHugh-Nagumo oscillators. The top panels depict the inter-spike intervals, bottom ones the output voltages of the oscillators.

cillator. The phase resetting curve corresponding to such the pulse is depicted in Fig. 3(a).

In the experiment, we started from random initial conditions and traced the dynamics of the output voltages and the inter-spike intervals of all the oscillators. We were able to detect all the regimes predicted theoretically, except for the global synchrony (Fig. 7(a)). The presumable reason of not finding this regime is its low stability and high sensitivity to the frequency detuning. In Fig. 7 the examples of two distinct jittering rotating waves are given.

We have demonstrated jittering rotating waves in the ring of pulse-coupled oscillators with no coupling delays. Previously, we have shown that jittering solutions emerge in the oscillator with delayed feedback generically, and the only necessary condition for that is the presence of parts with slope $<-1$ in the oscillator's PRC. In this case the appropriate choice of the delay allows to establish a jittering solution of arbitrary long period. Further we will show that jittering waves generically appear in rings 
of oscillators with instantaneous pulse coupling.

\section{Generic appearance of jittering waves in rings without delays}

In this section we show that under the assumption that one delayed pulse coupled oscillator (18) has a jittering regime, it is always possible to select a large enough number $N$ of oscillators in a ring (20) without delays such that this ring possesses a jittering wave.

As shown in [51], the branch of regular spiking solutions can be determined parametrically as $T(\psi), \tau(\psi)$, where

$$
\begin{aligned}
T(\psi) & =1-Z(\psi), \\
\tau(\psi) & =P(1-Z(\psi))+\psi,
\end{aligned}
$$

$P \in Z, T(\psi)$ is the inter-spike interval at time-delay $\tau(\psi)$ The stability of these regular spiking solutions is given by the condition $-1<\alpha<1 / P$, where $\alpha:=Z^{\prime}(\psi)$. For $\alpha=1 / P$, the regular solutions undergo a fold bifurcation, and for $\alpha=-1$ the multi-jitter bifurcation. Jittering solutions branch from the regular ones at the multi-jitter bifurcation points, i.e. at $\tau\left(\psi^{*}\right)=P\left(1-Z\left(\psi^{*}\right)\right)+\psi^{*}$, where $Z^{\prime}\left(\psi^{*}\right)=-1$. Each jittering regime consists of distinct inter-spike intervals $\Theta_{j}$ that form a periodic sequence. This sequence has a length $P+1$, thus the period of the jittering solution equals $T=\Theta_{1}+\Theta_{2}+\ldots+\Theta_{P+1}$. At the bifurcation point, all the inter-spike intervals tend to the period of the regular solution from which they have branched. Since the period of the regular solution equals $T_{R}=1-Z\left(\psi^{*}\right)$, the period of the emergent jittering solution at the bifurcation point equals $T_{J}=(P+1)\left(1-Z\left(\psi^{*}\right)\right)$.

Easy to show that at the bifurcation point the period of the emergent jittering solution $T$ is larger than the delay $\tau$. Each branch of jittering solutions connect two points of multi-jitter bifurcations A and B. Assume without loss of generality that $\tau_{A} / T_{A}<\tau_{B} / T_{B}<1$. Then it is always possible to select a rational number $M / N \in I=\left[\tau_{A} / T_{A}, \tau_{B} / T_{B}\right]$ so that the line $\tau / T=M / N$ intersects the branch. This would assure that the corresponding jittering solution can be embedded as a jittering rotating wave in the ring of $N$ oscillators with wave number $M$ (see (16)).

Thus, we have shown that jittering rotating waves generically emerge in a rings with instantaneous pulse coupling. It is always possible to take a sufficiently large number of oscillators and obtain a wave with an arbitrary large period. Let us estimate the minimal size $N$ of the ring which is necessary to embed the jittering solution. Substituting the expressions for the bifurcation parameters $\tau_{A, B}$ and $T_{A, B}$ one obtains

$$
I=\left[1-\frac{f\left(\psi_{A}\right)}{(P+1)}, 1-\frac{f\left(\psi_{B}\right)}{(P+1)}\right],
$$

where $f(\varphi)=1-\varphi /(1-Z(\varphi))$. As $P$ grows, the interval $I$ shrinks and approaches unity. From this observation it is easy to see that the minimal size $N$ is proportional to the jittering period $P$. Moreover, for large $P$ the wave number $M$ must be close to $N$.

\section{CONCLUSIONS}

In this paper, we have studied the relation between the dynamics of a single oscillator with delayed feedback and a feed-forward ring of identical oscillators. The obtained results may be summarized as follows:

1. Periodic solutions of the single oscillator appear as rotating waves in the ring with coupling delays $\sigma=$ $\tau-M T / N+k T$. Here, $k \in \mathbb{Z}$ and $M=0, \ldots, N-1$ is the wave-number of the rotating wave.

2. In particular, when the period is rationally related to the delay such as $\tau=M T / N$, the periodic solution embeds as a wave in the ring with instantaneous coupling $\sigma=0$.

3. The stability of the rotation waves in the ring for large delays $\sigma$ is equal to the stability of the corresponding periodic solution in the single oscillator for large delays $\tau$.

4. For large number $N$ of units in the ring, the stability of the rotation wave for $\sigma=0$ coincides with the stability of the corresponding periodic solution for $\tau \rightarrow \infty$. For small $N$, stability of the periodic solution for $\tau \rightarrow \infty$ is a necessary condition for the stability of the wave for $\sigma=0$.

The discovered relations between the dynamics of a single oscillator with delayed feedback and a ring of oscillators are of great interest. Particularly, it suggests that dynamical regimes observed in systems with delays may be also observed in systems without delays but with a ring topology. As an example we considered highperiodical jittering regimes in oscillators with pulse delayed feedback. We show that these regimes generically can be embedded as jittering rotating waves in rings of pulse-coupled oscillators without coupling delays.

\section{ACKNOWLEDGMENTS}

V.K., D.S., S.Y. and V.N. acknowledge the support from the Russian Scientific Foundation (project 16-4201043 for the Institute of Applied Physics) and the German Research Foundation (project SCHO 307/15-1 and YA 225/3-1 for TU Berlin). 
[1] M. C. Mackey, and L. Glass. Oscillation and chaos in physiological control systems. Science, 197, 287 (1977).

[2] M.C. Mackey. Periodic auto-immune hemolytic anemia: an induced dynamical disease. Bulletin of Mathematical Biology, 41(6), 829-834 (1979).

[3] R. Lang and K. Kobayashi, External optical feedback effects on semiconductor injection laser properties, IEEE J. Quantum Electron. 16, pp. 347-355 (1980).

[4] A. Longtin, and J.G. Milton. Modelling autonomous oscillations in the human pupil light reflex using non-linear delay-differential equations. Bulletin of Mathematical Biology, 51(5), 605-624 (1989).

[5] G. Giacomelli and A. Politi, Multiple scale analysis of delayed dynamical systems, Physica D 117, pp. 26-42 (1998).

[6] M. Wolfrum, S. Yanchuk, P. Hövel, and E. Schöll. Complex dynamics in delay-differential equations with large delay, Eur. Phys. J. Special Topics 191, pp. 91-103 (2010).

[7] M. C. Soriano, J. Garcia-Ojalvo, C. R. Mirasso, and I. Fischer, Complex photonics: Dynamics and applications of delay-coupled semiconductors lasers, Rev. Mod. Phys. 85, pp. 421-470 (2013).

[8] L. Larger, B. Penkovsky, and Yu. Maistrenko, Virtual chimera states for delayed-feedback systems, Phys. Rev. Lett. 111(5), 54103 (2013).

[9] L. Larger, M. C. Soriano, D. Brunner, L. Appeltant, J. M. Gutierrez, L. Pesquera, C. R. Mirasso, and I. Fischer, Photonic information processing beyond Turing: an optoelectronic implementation of reservoir computing, Opt. Express 20(3), pp. 3241-3249 (2012).

[10] M. Wolfrum and S. Yanchuk, Eckhaus instability in systems with large delay, Phys. Rev. Lett. 96, 220201 (2006).

[11] S. Yanchuk and G. Giacomelli, Spatio-temporal phenomena in complex systems with time delays, J. Phys. A 50(10), 103001 (2017).

[12] Y. Kuramoto and D. Battogtokh, Coexistence of coherence and incoherence in nonlocally coupled phase oscillators, Nonlinear Phenom. Complex Syst. 5(4), pp. 380-385 (2002).

[13] A. Pikovsky, M. Rosenblum, and J. Kurths, Synchronization. A universal concept in nonlinear sciences, Cambridge University Press (2001).

[14] F. A. Rodrigues, T. K. DM. Peron, P. Ji, and J. Kurths, The Kuramoto model in complex networks, Phys. Rep. 610, pp. 1-98 (2016).

[15] S. Boccaletti, V. Latora, Y. Moreno, M. Chavez, and D.U. Hwanga. Complex networks: Structure and dynamics, Phys. Rep. 424, pp. 175-308 (2006).

[16] J. Foss and J. Milton. Multistability in recurrent neural loops arising from delay. J Neurophysiol, 84(2), 975-985. Retrieved from http://www.ncbi.nlm.nih.gov/pubmed/10938321 (2000).

[17] X.-J. Wang. Synaptic reverberations underlying mnemonic persistent activity. Trends in Neurosciences, 24(8), 455-463. http://doi.org/10.1016/S01662236(00)01868-3 (2001).

[18] O. Sporns, and R. Kötter. Motifs in brain networks. PLoS Biology, 2(11), e369 (2004).

[19] J. Ma, and J. Wu. Multistability in spiking neuron models of delayed recurrent inhibitory loops. Neural Compu- tation, 19(8), 2124-2148 (2007).

[20] J. Ma, and J. Wu. Patterns, Memory and Periodicity in Two-Neuron Delayed Recurrent Inhibitory Loops. Mathematical Modelling of Natural Phenomena, 5(2), 67-99. http://doi.org/10.1051/mmnp/20105203 (2010).

[21] R. Milo, S. Shen-Orr, S. Itzkovitz, N. Kashtan, D. Chklovskii, and U. Alon. Network Motifs: Simple Building Blocks of Complex Networks. Science, 298(5594), 824-827. http://doi.org/10.1126/science.298.5594.824 (2002).

[22] U. Alon. Network motifs: theory and experimental approaches. Nature Reviews. Genetics, 8(6), 450-61. http://doi.org/10.1038/nrg2102 (2007).

[23] I. Kanter, M. Zigzag, A. Englert, F. Geissler, and W. Kinzel. Synchronization of unidirectional time delay chaotic networks and the greatest common divisor. EPL (Europhysics Letters), 93, 60003 (2011).

[24] J. T. Lizier, , F.M. Atayand J. Jost. Information storage, loop motifs, and clustered structure in complex networks. Physical Review E - Statistical, Nonlinear, and Soft Matter Physics, 86(2), 1-5. http://doi.org/10.1103/PhysRevE.86.026110 (2012).

[25] L. Lücken, J.P. Pade, K. Knauer, and S. Yanchuk. Reduction of interaction delays in networks. EPL (Europhysics Letters), 103, 10006 (2013).

[26] M. Bonnin, Waves and patterns in ring lattices with delays, Phys. D 238(1), pp. 77-87 (2009).

[27] P. Perlikowski, S. Yanchuk, O. V. Popovych, and P. A. Tass, Periodic patterns in a ring of delay-coupled oscillators, Phys. Rev. E 82(3), 036208 (2010).

[28] S. Bungay and S.A. Campbell, Patterns of oscillation in a ring of identical cells with delayed coupling, Int. J. Bifurcation and Chaos 17, pp. 3109-3125 (2007).

[29] I. P. Marino, V. Pérez-Mu nuzuri, V. Pérez-Villar, E. Sánchez, and M. A. Matías, Interaction of chaotic rotating waves in coupled rings of chaotic cells, Phys. D 128(2-4), pp. 224-235 (1999).

[30] Y. Zhang, G. Hu, and H. A. Cerdeira, How does a periodic rotating wave emerge from high-dimensional chaos in a ring of coupled chaotic oscillators?, Phys. Rev. E 64(3), 037203 (2001).

[31] O. D'Huys, I. Fischer, J. Danckaert and R. Vicente, Spectral and correlation properties of rings of delay-coupled elements: Comparing linear and nonlinear systems, Phys. Rev. E 85, 056209 (2012).

[32] Y. Horikawa and H. Kitajima, Duration of transient oscillations in ring networks of unidirectionally coupled neurons, Phys. D 238(2), pp. 216-225 (2009).

[33] Y. Horikawa and H. Kitajima, Transient chaotic rotating waves in a ring of unidirectionally coupled symmetric Bonhoeffer-van der Pol oscillators near a codimensiontwo bifurcation point, Chaos 22(3), 033115 (2012).

[34] R. Dodla, A. Sen, and G. L. Johnston, Phase-locked patterns and amplitude death in a ring of delay-coupled limit cycle oscillators, Phys. Rev. E 69(5), 056217 (2004).

[35] M. Kantner and S. Yanchuk, Bifurcation analysis of delay-induced patterns in a ring of Hodgkin-Huxley neurons, Phil. Trans. Roy. Soc. A 371, 20120470 (2013).

[36] G. Van der Sande, M. C. Soriano, I. Fischer, and C. R. Mirasso, Dynamics, correlation scaling, and synchronization behavior in rings of delay-coupled oscillators, Phys. 
Rev. E 77(5), 055202 (2008).

[37] O. D'Huys, R. Vicente, T. Erneux, J. Danckaert, and I. Fischer, Synchronization properties of network motifs: Influence of coupling delay and symmetry, Chaos 18(3), 037116 (2008).

[38] E. Sánchez and M. A. Matías, Experimental observation of a periodic rotating wave in rings of unidirectionally coupled analog Lorenz oscillators, Phys. Rev. E 57(5), pp. 6184-6186 (1998).

[39] C. R. S. Williams, F. Sorrentino, T. E. Murphy, and R. Roy, Synchronization states and multistability in a ring of periodic oscillators: Experimentally variable coupling delays, Chaos 23, 043117 (2013).

[40] P. Woafo and H. G. Enjieu Kadji, Synchronized states in a ring of mutually coupled self-sustained electrical oscillators, Phys. Rev. E 69(4), 046206 (2004).

[41] V. Klinshov, D. Shchapin, L. Lücken, S. Yanchuk, and V. Nekorkin, Experimental study of jittering chimeras in a ring of excitable units, AIP Conference Proceedings 1738, 210007 (2016).

[42] D. P. Rosin, D. Rontani, D. J. Gauthier, and E. Schöll, Control of synchronization patterns in neural-like Boolean networks, Phys. Rev. Lett. 110, 104102 (2013).

[43] O. D'Huys, J. Lohmann, N. D. Haynes, and D.J. Gauthier, Super-transient scaling in time-delay autonomous Boolean network motifs, Chaos 26, 094810 (2016).

[44] J. Lohmann, O. D'Huys, N.D. Haynes, E. Schöll and D.J. Gauthier, Transient dynamics and their control in timedelay autonomous Boolean ring networks, Phys. Rev. E 95, 022211 (2017).

[45] M. Lichtner, M. Wolfrum, and S. Yanchuk, The spectrum of delay differential equations with large delay, SIAM J. Math. Anal. 43, pp. 788-802 (2011).

[46] J. Sieber, M. Wolfrum, M. Lichtner, and S. Yanchuk, On the stability of periodic orbits in delay equations with large delay, Discrete Contin. Dyn. Syst. A 33, pp. 3109-3134 (2013).
[47] S. Yanchuk and P. Perlikowski, Delay and periodicity, Phys. Rev. E 79(4), 046221 (2009).

[48] P. Perlikowski, S. Yanchuk, M. Wolfrum, A. Stefanski, P. Mosiolek, and T. Kapitaniak, Routes to complex dynamics in a ring of unidirectionally coupled systems, Chaos 20, 013111 (2010).

[49] S. Yanchuk and M. Wolfrum, Destabilization patterns in chains of coupled oscillators, Phys. Rev. E 77(2), 026212 (2008).

[50] G. Van der Sande, M. C. Soriano, I. Fischer, and C. R. Mirasso, Dynamics, correlation scaling, and synchronization behavior in rings of delay-coupled oscillators, Phys. Rev. E 77, 055202 (2008).

[51] V. Klinshov, L. Lücken, D. Shchapin, V. Nekorkin, and S. Yanchuk, Multistable jittering in oscillators with pulsatile delayed feedback, Phys. Rev. Lett. 114, 178103 (2015).

[52] V. Klinshov, L. Lücken, D. Shchapin, V. Nekorkin, and S. Yanchuk, Emergence and combinatorial accumulation of jittering regimes in spiking oscillators with delayed feedback, Phys. Rev. E 92, 042914 (2015).

[53] V. Klinshov, D. Shchapin, S. Yanchuk, and V. Nekorkin, Jittering waves in rings of pulse oscillators, Phys. Rev. E 94(1), 012206 (2016).

[54] O. D'Huys, T. Jüngling, and W. Kinzel, Stochastic switching in delay-coupled oscillators, Phys. Rev. E 90(3), 032918 (2014).

[55] O. D'Huys, T. Jüngling, and W. Kinzel, On the interplay of noise and delay in coupled oscillators, In: E. Schöll, S. Klapp, and P. Hövel (eds.), Control of self-organizing nonlinear systems, Springer Complexity (2016).

[56] G. Giacomelli and A. Politi, Relationship between delayed and spatially extended dynamical systems, Phys. Rev. Lett. 76(15), pp. 2686-2689 (1996).

[57] D. S. Shchapin, J. Communicat. Technology Electronics 54175 (2009).

[58] V.V. Klinshov, D.S. Shchapin, and V.I. Nekorkin. Cross-frequency synchronization of oscillators with timedelayed coupling. Phys. Rev. E, 90:042923, (2014). 